\title{
On the estimation of density-weighted average derivative by wavelet methods under various dependence structures
}

\author{
Christophe Chesneau $^{1}$, Maher Kachour ${ }^{3}$ and Fabien Navarro ${ }^{1,2}$ \\ ${ }^{1}$ LMNO, Université de Caen Basse-Normandie, Département de Mathématiques, \\ UFR de Sciences, 14032 Caen Cedex, France. E-mail: christophe.chesneau@unicaen.fr. \\ ${ }^{2}$ GREYC CNRS-ENSICAEN-Université de Caen, \\ 14050 Caen Cedex, France. E-mail: fabien.navarro@ensicaen.fr. \\ ${ }^{3}$ École supérieure de commerce IDRAC, 47, rue Sergent Michel Berthet CP 607, \\ 69258 Lyon Cedex 09, France. E-mail: maher.kachour@idraclyon.com.
}

May 30, 2013

\begin{abstract}
The problem of estimating the density-weighted average derivative of a regression function is considered. We present a new consistent estimator based on a plug-in approach and wavelet projections. Its performances are explored under various dependence structures on the observations: the independent case, the $\rho$-mixing case and the $\alpha$-mixing case. More precisely, denoting $n$ the number of observations, in the independent case, we prove that it attains $1 / n$ under the mean squared error, in the $\rho$-mixing case, $1 / \sqrt{n}$ under the mean absolute error, and, in the $\alpha$-mixing case, $\sqrt{\ln n / n}$ under the mean absolute error. A short simulation study illustrates the theory.
\end{abstract}

Key words and phrases: Nonparametric estimation of density-weighted average derivative, 'Plug-in' approach, Wavelets, Consistency, $\rho$-mixing, $\alpha$-mixing.

AMS 2000 Subject Classifications: 62G07, 62G20.

\section{Introduction}

We consider the following nonparametric regression model:

$$
Y_{i}=f\left(X_{i}\right)+\xi_{i}, \quad i \in\{1, \ldots, n\},
$$


where the design variables (or input variables) $X_{1}, \ldots, X_{n}$ are $n$ identically distributed random variables with common unknown density function $g$, the noise $\xi_{1}, \ldots, \xi_{n}$ are $n$ identically distributed random variables with $\mathbb{E}\left(\xi_{1}\right)=0$ and $\mathbb{E}\left(\xi_{1}^{4}\right)<\infty$, and $f$ is an unknown regression function. Moreover, it is understood that $\xi_{i}$ is independent of $X_{i}$, for any $i \in\{1, \ldots, n\}$. In this paper, we are interested in the pointwise estimation of the density-weighted average derivative, which is defined as follows

$$
\delta=\mathbb{E}\left(g\left(X_{1}\right) f^{\prime}\left(X_{1}\right)\right)=\int g^{2}(x) f^{\prime}(x) d x,
$$

from $\left(X_{1}, Y_{1}\right), \ldots,\left(X_{n}, Y_{n}\right)$. It is known that the estimation of $\delta$ is of interest in many statistical and econometric models, especially in the context of estimation of coefficients in index models (for review see, e.g., Powell (1994) and Matzkin (2007)). Indeed, estimation of coefficients in single index models relies on the fact that averaged derivatives of the conditional mean are proportional to the coefficients (see, e.g., Stoker $(1986,1989)$, Powell et al. (1989) and Härdle and Stoker (1989)). Also further motivation of average derivative estimate can be found in specific problems in economics, such as measuring the positive definiteness of the aggregate income effects matrix for assessing the "Law of Demand" (see Härdle et al. (1991)), policy analysis of tax and subsidy reform (see Deaton and $\mathrm{Ng}(1998)$ ), and nonlinear pricing in labor markets (see Coppejans and Sieg (2005)).

When $\left(X_{1}, Y_{1}\right), \ldots,\left(X_{n}, Y_{n}\right)$ are i.i.d., the most frequently used nonparametric techniques are based on kernel estimators. Three different approaches can be found in Härdle and Stoker (1989), Powell et al. (1989) and Stoker (1991). Their consistency are established. Recent theoretical and practical developments related to these estimators can be found in, e.g., Härdle et al. (1992), Türlach (1994), Powell and Stoker (1996), Banerjee (2007), Schafgans and Zinde-Walsh (2010) and Cattaneo et al. (2010, 2011). A new estimator based on orthogonal series methods has been introduced in Prakasa Rao (1995). More precisely, using the same plug-in approach of Powell et al. (1989), $\hat{\delta}$ the estimator of the density-weighted average derivative has the following form

$$
\hat{\delta}=-\frac{2}{n} \sum_{i=1}^{n} Y_{i} \hat{g}_{i}^{\prime}\left(X_{i}\right),
$$

where $\hat{g}_{i}^{\prime}$ denotes an orthogonal series estimator of $g^{\prime}$ constructed from $X_{1}, \ldots, X_{i-1}, X_{i+1}, X_{n}$. Moreover, the consistency of this estimator is proved.

In this study, we develop a new estimator based on a different plug-in approach to the one in Powell et al. (1989) and a particular orthogonal series method: the wavelet series method. The main advantage of this method is its adaptability to the varying degrees of smoothness of the underlying unknown curves. For a complete discussion of wavelets and their applications in statistics, we refer to Antoniadis (1997), Härdle et al. (1998) and Vidakovic (1999). 
When $\left(X_{1}, Y_{1}\right), \ldots,\left(X_{n}, Y_{n}\right)$ are i.i.d., we prove that our estimator attains the parametric rate of convergence $1 / n$ under the Mean Square Error (MSE). This rate is a bit better to the one attains by the estimator in Prakasa Rao (1995). Moreover, the flexibility of our approach enables us to consider possible dependent observations, thus opening new perspectives of applications. This is illustrated by the considerations of the $\rho$-mixing dependence introduced by Kolmogorov and Rozanov (1960) and the $\alpha$-mixing dependence introduced by Rosenblatt (1956). Adopting the Mean Absolute Error (MAE), we prove that our estimator attains the rate of convergence $1 / \sqrt{n}$ in the $\rho$-mixing case, and $\sqrt{\ln n / n}$ in the $\alpha$-mixing case. All these results prove the consistency of our estimator and its robustness in term of dependence on the observations. Mention that, to the best of our knowledge, the estimation of $\delta$ in such a dependent setting has never been explored earlier. A simulation study illustrates the performance of the proposed wavelet method in finite sample situations.

The remainder of the paper is set out as follows. Next, in Section 2, we discuss the preliminaries of the wavelet orthogonal bases and we recall the definition of some mixing conditions. Section is devoted to our wavelet estimator. Assumptions on (1.1) are described in Section 4. Section 5 presents our main theoretical results. A short simulation study illustrates the theory in Section 6. Finally, the proofs are postponed to Section 7.

\section{Preliminaries and Definitions}

\subsection{Orthonormal bases of compactly supported wavelets}

Let the following set of functions

$$
\mathbb{L}^{2}([0,1])=\left\{h:[0,1] \rightarrow \mathbb{R} ;\|h\|_{2}^{2}=\int_{0}^{1}(h(x))^{2} d x\right\} .
$$

For the purposes of this paper, we use the compactly supported wavelet bases on $[0,1]$ briefly described below.

Let $N \geq 10$ be a fixed integer, and $\phi$ and $\psi$ be the initial wavelet functions of the Daubechies wavelets $d b 2 N$. These functions have the features to be compactly supported and $\mathcal{C}^{1}$ (see Daubechies (1992)). Set

$$
\phi_{j, k}(x)=2^{j / 2} \phi\left(2^{j} x-k\right), \quad \psi_{j, k}(x)=2^{j / 2} \psi\left(2^{j} x-k\right)
$$

and $\Lambda_{j}=\left\{0, \ldots, 2^{j}-1\right\}$. Then, with an appropriate treatment at the boundaries, the collection

$$
\mathcal{B}=\left\{\phi_{\tau, k}, k \in \Lambda_{\tau} ; \psi_{j, k} ; j \in \mathbb{N}-\{0, \ldots, \tau-1\}, k \in \Lambda_{j}\right\}
$$

is an orthonormal basis of $\mathbb{L}^{2}([0,1])$, provided the primary resolution level $\tau$ is large enough to ensure that the support of $\phi_{\tau, k}$ and $\psi_{\tau, k}$ with $k \in \Lambda_{\tau}$ is not the whole of $[0,1]$ (see, e.g., Cohen et al. (1993) and Mallat (2009)). 
Hence, any $h \in \mathbb{L}^{2}([0,1])$ can be expanded on $\mathcal{B}$ as

$$
h(x)=\sum_{k \in \Lambda_{\tau}} \alpha_{\tau, k} \phi_{\tau, k}(x)+\sum_{j=\tau}^{\infty} \sum_{k \in \Lambda_{j}} \beta_{j, k} \psi_{j, k}(x),
$$

where

$$
\alpha_{\tau, k}=\int_{0}^{1} h(x) \phi_{\tau, k}(x) d x, \quad \beta_{j, k}=\int_{0}^{1} h(x) \psi_{j, k}(x) d x .
$$

For more details about wavelet bases, we refer to Meyer (1992), Daubechies (1992), Cohen et al. (1993) and Mallat (2009).

\subsection{Mixing conditions}

In this subsection, we recall the definitions of two standard kinds of dependence for random sequences: the $\rho$-mixing dependence and the $\alpha$-mixing dependence.

Let $Z=\left(Z_{t}\right)_{t \in \mathbb{Z}}$ be a strictly stationary random sequence defined on a probability space $(\Omega, \mathcal{A}, \mathbb{P})$. For $j \in \mathbb{Z}$, define the $\sigma$-fields

$$
\mathcal{F}_{-\infty, j}^{Z}=\sigma\left(Z_{k}, k \leq j\right), \quad \mathcal{F}_{j, \infty}^{Z}=\sigma\left(Z_{k}, k \geq j\right) .
$$

Definition 2.1 ( $\rho$-mixing dependence) For any $m \in \mathbb{Z}$, we define the $m$-th maximal correlation coefficient of $\left(Z_{t}\right)_{t \in \mathbb{Z}}$ by

$$
\rho_{m}=\sup _{(U, V) \in \mathbb{L}^{2}\left(\mathcal{F}_{-\infty, 0}^{Z}\right) \times \mathbb{L}^{2}\left(\mathcal{F}_{m, \infty}^{Z}\right)} \frac{|\operatorname{Cov}(U, V)|}{\sqrt{\mathbb{V}(U) \mathbb{V}(V)}},
$$

where $\operatorname{Cov}(.,$.$) denotes the covariance function and \mathbb{L}^{2}(\mathcal{D})$ denotes the space of squareintegrable, $\mathcal{D}$-measurable (real-valued) random variables for any $\mathcal{D} \in\left\{\mathcal{F}_{-\infty, 0}^{Z}, \mathcal{F}_{m, \infty}^{Z}\right\}$.

We say that $\left(Z_{t}\right)_{t \in \mathbb{Z}}$ is $\rho$-mixing if and only if $\lim _{m \rightarrow \infty} \rho_{m}=0$.

Full details on $\rho$-mixing can be found in, e.g., Kolmogorov and Rozanov (1960), Doukhan (1994), Shao (1995) and Zhengyan and Lu (1996).

Definition 2.2 ( $\alpha$-mixing dependence) For any $m \in \mathbb{Z}$, we define the $m$-th strong mixing coefficient of $\left(Z_{t}\right)_{t \in \mathbb{Z}}$ by

$$
\alpha_{m}=\sup _{(A, B) \in \mathcal{F}_{-\infty, 0}^{Z} \times \mathcal{F}_{m, \infty}^{Z}}|\mathbb{P}(A \cap B)-\mathbb{P}(A) \mathbb{P}(B)| .
$$

We say that $\left(Z_{t}\right)_{t \in \mathbb{Z}}$ is $\alpha$-mixing if and only if $\lim _{m \rightarrow \infty} \alpha_{m}=0$.

Full details on $\alpha$-mixing can be found in, e.g., Rosenblatt (1956), Doukhan (1994), Carrasco and Chen (2002) and Fryzlewicz and Subba Rao (2011). 


\section{A new wavelet-based estimator for $\delta$}

Proposition 3.1 below provides another expression of the density-weighted average derivative (1.2) in terms of wavelet coefficients.

Proposition 3.1 Consider the regression model with random design (1.1). Suppose that $\operatorname{supp}\left(X_{1}\right)=[0,1], f g \in \mathbb{L}^{2}([0,1]), g^{\prime} \in \mathbb{L}^{2}([0,1])$ and $g(0)=g(1)=0$. Then the densityweighted average derivative (1.2) can be expressed as

$$
\delta=-2\left(\sum_{k \in \Lambda_{\tau}} \alpha_{\tau, k} c_{\tau, k}+\sum_{j=\tau}^{\infty} \sum_{k \in \Lambda_{j}} \beta_{j, k} d_{j, k}\right),
$$

where

$$
\begin{array}{ll}
\alpha_{\tau, k}=\int_{0}^{1} f(x) g(x) \phi_{\tau, k}(x) d x, & c_{\tau, k}=\int_{0}^{1} g^{\prime}(x) \phi_{\tau, k}(x) d x, \\
\beta_{j, k}=\int_{0}^{1} f(x) g(x) \psi_{j, k}(x) d x, & d_{j, k}=\int_{0}^{1} g^{\prime}(x) \psi_{j, k}(x) d x .
\end{array}
$$

We consider the following plug-in estimator for $\delta$ :

$$
\hat{\delta}=-2\left(\sum_{k \in \Lambda_{\tau}} \hat{\alpha}_{\tau, k} \hat{c}_{\tau, k}+\sum_{j=\tau}^{j_{0}} \sum_{k \in \Lambda_{j}} \hat{\beta}_{j, k} \hat{d}_{j, k}\right),
$$

where

$$
\begin{aligned}
\hat{\alpha}_{\tau, k} & =\frac{1}{n} \sum_{i=1}^{n} Y_{i} \phi_{\tau, k}\left(X_{i}\right), & \hat{c}_{\tau, k} & =-\frac{1}{n} \sum_{i=1}^{n}\left(\phi_{\tau, k}\right)^{\prime}\left(X_{i}\right), \\
\hat{\beta}_{j, k} & =\frac{1}{n} \sum_{i=1}^{n} Y_{i} \psi_{j, k}\left(X_{i}\right), & \hat{d}_{j, k} & =-\frac{1}{n} \sum_{i=1}^{n}\left(\psi_{j, k}\right)^{\prime}\left(X_{i}\right)
\end{aligned}
$$

and $j_{0}$ is an integer which will be chosen a posteriori.

Remark 3.1 The construction of our estimator (3.3) uses a plug-in approach derived to Proposition 3.1. Note that it completely differs to the estimator (1.3) of Prakasa Rao (1995).

Remark 3.2 Mention that $\hat{c}_{\tau, k}$ (3.4) and $\hat{d}_{j, k}$ (3.5) have been introduced by Prakasa Rao (1996) in the derivative density estimation problem via wavelets. In the context of dependent observations, see Chaubey et al. (2005) and Chaubey et al. (2006). 
Proposition 3.2 Suppose that $\operatorname{supp}\left(X_{1}\right)=[0,1]$. Then

- $\hat{\alpha}_{\tau, k}$ (3.4) and $\hat{\beta}_{j, k}$ (3.5) are unbiased estimators for $\alpha_{\tau, k}$ (3.1) and $\beta_{j, k}$ (3.2) respectively.

- under $g(0)=g(1)=0, \hat{c}_{\tau, k}$ (3.4) and $\hat{d}_{j, k}(3.5)$ are unbiased estimators for $c_{\tau, k}(3.1)$ and $d_{j, k}(3.2)$ respectively.

\section{Model assumptions}

\subsection{Assumptions on $f$ and $g$}

We formulate the following assumptions on $f$ and $g$ :

H1. The support of $X_{1}$, denoted by $\operatorname{supp}\left(X_{1}\right)$, is compact. In order to fix the notations, we suppose that $\operatorname{supp}\left(X_{1}\right)=[0,1]$.

H2. There exists a known constant $C_{1}>0$ such that

$$
\sup _{x \in[0,1]}|f(x)| \leq C_{1}
$$

H3. The function $g$ satisfies $g(0)=g(1)=0$ and there exist two known constants $C_{2}>0$ and $C_{3}>0$ such that

$$
\sup _{x \in[0,1]} g(x) \leq C_{2}, \quad \sup _{x \in[0,1]}\left|g^{\prime}(x)\right| \leq C_{3} .
$$

Let us now make some brief comments on these assumptions. The assumption $\mathbf{H 1}$ is similar to (Härdle and Tsybakov, 1993, Assumption (A3)) or (Banerjee, 2007, Assumption A1). In our study, we make it to apply the wavelet methodology described in Section 3. The noncompactly supported case arises several technical difficulties for the wavelet methods (see Juditsky and Lambert-Lacroix (2004) and Reynaud-Bouret et al. (2011)). Their adaptations in the context of the density-weighted average derivative estimation is not immediatly clear. The assumptions $\mathbf{H 2}$ and $\mathbf{H 3}$ are standard in this framework. They are satisfied by a wide variety of functions.

\subsection{Assumptions on the wavelet coefficients of $f g$ and $g^{\prime}$}

Let $s_{1}>0, s_{2}>0$ and $\beta_{j, k}$ and $d_{j, k}$ be given by (3.2). We formulate the following assumptions on $\beta_{j, k}$ and $d_{j, k}$ : 
H4 $\left(s_{1}\right)$. There exists a constant $C_{4}>0$ such that

$$
\left|\beta_{j, k}\right| \leq C_{4} 2^{-j\left(s_{1}+1 / 2\right)} .
$$

$\mathbf{H 5}\left(s_{2}\right)$. There exists a constant $C_{5}>0$ such that

$$
\left|d_{j, k}\right| \leq C_{5} 2^{-j\left(s_{2}+1 / 2\right)} .
$$

The assumptions $\mathbf{H 4}\left(s_{1}\right)$ and $\mathbf{H 5}\left(s_{2}\right)$ characterize the degrees of smoothness of $f g$ and $g^{\prime}$ respectively.

Remark 4.1 In terms of function sets, $\mathbf{H 4}\left(s_{1}\right)$ and $\mathbf{H 5}\left(s_{2}\right)$ are equivalent to $f g \in \mathcal{L}_{s_{1}}\left(M_{1}\right)$ and $g^{\prime} \in \mathcal{L}_{s_{2}}\left(M_{2}\right)$ with $M_{1}>0$ and $M_{2}>0$ respectively, where

$$
\mathcal{L}_{s}(M)=\left\{h:[0,1] \rightarrow \mathbb{R} ;\left|h^{(\lfloor s\rfloor)}(x)-h^{(\lfloor s\rfloor)}(y)\right| \leq M|x-y|^{\alpha}, s=\lfloor s\rfloor+\alpha, \alpha \in(0,1]\right\},
$$

$M>0,\lfloor s\rfloor$ is the integer part of $s$ and $h^{(\lfloor s\rfloor)}$ the $\lfloor s\rfloor$-th derivatives of $h$. We refer to (Härdle et al., 1998, Chapter 8).

\section{Main results}

\subsection{The independent case}

In this subsection, we suppose that $\left(X_{1}, Y_{1}\right), \ldots,\left(X_{n}, Y_{n}\right)$ are independent.

Before presenting the main result, let us set two propositions which will be usefull in the proofs.

Proposition 5.1 Consider the nonparametric regression model, defined by (1.1). Assume that H1, $\mathbf{H 2}$ and $\mathbf{H 3}$ hold. Let $\beta_{j, k}$ and $d_{j, k}$ be given by (3.2), and $\hat{\beta}_{j, k}$ and $\hat{d}_{j, k}$ be given by (3.5) with $j$ such that $2^{j} \leq n$. Then

- there exists a constant $C>0$ such that

$$
\mathbb{E}\left(\left(\hat{\beta}_{j, k}-\beta_{j, k}\right)^{4}\right) \leq C \frac{1}{n^{2}},
$$

- there exists a constant $C>0$ such that

$$
\mathbb{E}\left(\left(\hat{d}_{j, k}-d_{j, k}\right)^{4}\right) \leq C \frac{2^{4 j}}{n^{2}}
$$

These inequalities hold with $\left(\hat{\alpha}_{\tau, k}, \hat{c}_{\tau, k}\right)$ in (3.4) instead of $\left(\hat{\beta}_{j, k}, \hat{d}_{j, k}\right)$, and $\left(\alpha_{\tau, k}, c_{\tau, k}\right)$ in $(3.1)$ instead of $\left(\beta_{j, k}, d_{j, k}\right)$ for $j=\tau$. 
Proposition 5.2 Consider the nonparametric regression model, defined by (1.1).

- Suppose that H1, H2, H3, H4 $\left(s_{1}\right)$ and $\mathbf{H 5}\left(s_{2}\right)$ hold. Let $\beta_{j, k}$ and $d_{j, k}$ be given by (3.2), and $\hat{\beta}_{j, k}$ and $\hat{d}_{j, k}$ be given by (3.5) with $j$ such that $2^{j} \leq n$. Then there exists a constant $C>0$ such that

$$
\mathbb{E}\left(\left(\hat{\beta}_{j, k} \hat{d}_{j, k}-\beta_{j, k} d_{j, k}\right)^{2}\right) \leq C\left(\frac{2^{-j\left(2 s_{1}-1\right)}}{n}+\frac{2^{-j\left(2 s_{2}+1\right)}}{n}+\frac{2^{2 j}}{n^{2}}\right) .
$$

- Suppose that H1, H2 and $\mathbf{H 3}$ hold. Let $\alpha_{\tau, k}$ and $c_{\tau, k}$ be given by (3.1), and $\hat{\alpha}_{\tau, k}$ and $\hat{c}_{\tau, k}$ be given by (3.4). Then there exists a constant $C>0$ such that

$$
\mathbb{E}\left(\left(\hat{\alpha}_{\tau, k} \hat{c}_{\tau, k}-\alpha_{\tau, k} c_{\tau, k}\right)^{2}\right) \leq C \frac{1}{n}
$$

The following theorem establishes the upper bound of the MSE of our estimator.

Theorem 5.1 Assume that H1, H2, H3, H4 $\left(s_{1}\right)$ with $s_{1}>3 / 2$ and $\mathbf{H 5}\left(s_{2}\right)$ with $s_{2}>1 / 2$ hold. Let $\delta$ be given by (1.2) and $\hat{\delta}$ be given by (3.3) with $j_{0}$ such that $n^{1 / 4}<2^{j_{0}+1} \leq 2 n^{1 / 4}$. Then there exists a constant $C>0$ such that

$$
\mathbb{E}\left((\hat{\delta}-\delta)^{2}\right) \leq C \frac{1}{n}
$$

Remark 5.1 Theorem 5.1 shows that, under some assumptions, our estimator (3.3) has a better MSE than the one in Prakasa Rao (1995), i.e. $q^{2}(n) / n$, where $q(n)$ satifies $\lim _{n \rightarrow \infty} q(n)=\infty$.

Remark 5.2 The level $j_{0}$ described in Theorem 5.1 is such that $\hat{\delta}$ attains the parametric rate of convergence $1 / n$ without depending on the knowledge of the regularity of $f$ or $g$ in its construction. In this sense, $\hat{\delta}$ is adaptive.

There are many practical situations in which it is not appropriate to assume that the observations $\left(X_{1}, Y_{1}\right), \ldots,\left(X_{n}, Y_{n}\right)$ are independent. The most typical scenario concerns the dynamic economic systems which are modelled as multiple time series. For details and applications of dependent nonparametric regression model (1.1), see White and Domowitz (1984), Lütkepohl (1992) and the references therein.

The rest of the study is devoted to the estimation of $\delta$ in the $\rho$-mixing case and the $\alpha$-mixing case. For technical convenience, the performance of (3.3) is explored via the MAE (not the MSE). 


\subsection{The $\rho$-mixing case}

Now, we assume that $\left(X_{1}, Y_{1}\right), \ldots,\left(X_{n}, Y_{n}\right)$ coming from a $\rho$-mixing strictly stationary process $\left(X_{t}, Y_{t}\right)_{t \in \mathbb{Z}}(1.1)$ (for details see Definition 2.1).

Before presenting the main result, let us set two propositions which will be usefull in the proofs.

Proposition 5.3 Consider the nonparametric regression model, defined by (1.1). Suppose that H1, H2, $\mathbf{H 3}$ and (5.5) hold. Let $\beta_{j, k}$ and $d_{j, k}$ be given by (3.2), and $\hat{\beta}_{j, k}$ and $\hat{d}_{j, k}$ be given by (3.5). Then

- there exists a constant $C>0$ such that

$$
\mathbb{E}\left(\left(\hat{\beta}_{j, k}-\beta_{j, k}\right)^{2}\right) \leq C \frac{1}{n},
$$

- there exists a constant $C>0$ such that

$$
\mathbb{E}\left(\left(\hat{d}_{j, k}-d_{j, k}\right)^{2}\right) \leq C \frac{2^{2 j}}{n} .
$$

These inequalities hold with $\left(\hat{\alpha}_{\tau, k}, \hat{c}_{\tau, k}\right)$ in (3.4) instead of $\left(\hat{\beta}_{j, k}, \hat{d}_{j, k}\right)$, and $\left(\alpha_{\tau, k}, c_{\tau, k}\right)$ in $(3.1)$ instead of $\left(\beta_{j, k}, d_{j, k}\right)$ for $j=\tau$.

Proposition 5.4 Consider the nonparametric regression model, defined by (1.1).

- Suppose that H1, H2, H3, H4 $\left(s_{1}\right), \mathbf{H 5}\left(s_{2}\right)$ and (5.5) hold, Let $\beta_{j, k}$ and $d_{j, k}$ be given by (3.2), and $\hat{\beta}_{j, k}$ and $\hat{d}_{j, k}$ be given by (3.5). Then there exists a constant $C>0$ such that

$$
\mathbb{E}\left(\left|\hat{\beta}_{j, k} \hat{d}_{j, k}-\beta_{j, k} d_{j, k}\right|\right) \leq C\left(\frac{2^{-j\left(s_{1}-1 / 2\right)}}{\sqrt{n}}+\frac{2^{-j\left(s_{2}+1 / 2\right)}}{\sqrt{n}}+\frac{2^{j}}{n}\right)
$$

- Suppose that H1, H2, H3 and (5.5) hold. Let $\alpha_{\tau, k}$ and $c_{\tau, k}$ be given by (3.1), and $\hat{\alpha}_{\tau, k}$ and $\hat{c}_{\tau, k}$ be given by (3.4). Then there exists a constant $C>0$ such that

$$
\mathbb{E}\left(\left|\hat{\alpha}_{\tau, k} \hat{c}_{\tau, k}-\alpha_{\tau, k} c_{\tau, k}\right|\right) \leq C \frac{1}{\sqrt{n}} .
$$

Theorem 5.2 determines the upper bound of the MAE of our estimator in the $\rho$-mixing case.

Theorem 5.2 Consider the nonparametric regression model, defined by (1.1). Suppose that 
- there exists a constant $C_{*}>0$ such that

$$
\sum_{m=1}^{\infty} \rho_{m} \leq C_{*},
$$

- H1, H2, H3, H4 $\left(s_{1}\right)$ with $s_{1}>3 / 2$ and $\mathbf{H 5}\left(s_{2}\right)$ with $s_{2}>1 / 2$ hold.

Let $\delta$ be given by (1.2) and $\hat{\delta}$ be given by (3.3) with $j_{0}$ such that $n^{1 / 4}<2^{j_{0}+1} \leq 2 n^{1 / 4}$. Then there exists a constant $C>0$ such that

$$
\mathbb{E}(|\hat{\delta}-\delta|) \leq C \frac{1}{\sqrt{n}}
$$

\subsection{The $\alpha$-mixing case}

Here, we assume that $\left(X_{1}, Y_{1}\right), \ldots,\left(X_{n}, Y_{n}\right)$ coming from a $\alpha$-mixing strictly stationary process $\left(X_{t}, Y_{t}\right)_{t \in \mathbb{Z}}(1.1)$ (for details see Definition 2.2).

Again, before presenting the main result, let us set two propositions which will be usefull in the proofs.

Proposition 5.5 Consider the nonparametric regression model, defined by (1.1). Suppose that

- there exist two constants $a>0$ and $b>0$ such that the strong mixing coefficient satisfies

$$
\alpha_{m} \leq a b^{-m}
$$

- H1, H2, H3, H4 $\left(s_{1}\right)$ with $s_{1}>3 / 2$ and $\mathbf{H 5}\left(s_{2}\right)$ with $s_{2}>1 / 2$ hold.

Let $\beta_{j, k}$ and $d_{j, k}$ be given by (3.2), and $\hat{\beta}_{j, k}$ and $\hat{d}_{j, k}$ be given by (3.5) with $j$ such that $2^{j} \leq n$. Then

- there exists a constant $C>0$ such that

$$
\mathbb{E}\left(\left(\hat{\beta}_{j, k}-\beta_{j, k}\right)^{2}\right) \leq C \frac{\ln n}{n},
$$

- there exists a constant $C>0$ such that

$$
\mathbb{E}\left(\left(\hat{d}_{j, k}-d_{j, k}\right)^{2}\right) \leq C \frac{2^{2 j} \ln n}{n}
$$

These inequalities hold with $\left(\hat{\alpha}_{\tau, k}, \hat{c}_{\tau, k}\right)$ in (3.4) instead of $\left(\hat{\beta}_{j, k}, \hat{d}_{j, k}\right)$, and $\left(\alpha_{\tau, k}, c_{\tau, k}\right)$ in $(3.1)$ instead of $\left(\beta_{j, k}, d_{j, k}\right)$ for $j=\tau$. 
Proposition 5.6 Consider the nonparametric regression model, defined by (1.1).

- Suppose that H1, H2, H3, H4 $\left(s_{1}\right), \mathbf{H 5}\left(s_{2}\right)$ and (5.6) hold. Let $\beta_{j, k}$ and $d_{j, k}$ be given by (3.2), and $\hat{\beta}_{j, k}$ and $\hat{d}_{j, k}$ be given by (3.5) with $j$ satisfying $2^{j} \leq n$. Then there exists a constant $C>0$ such that

$$
\mathbb{E}\left(\left|\hat{\beta}_{j, k} \hat{d}_{j, k}-\beta_{j, k} d_{j, k}\right|\right) \leq C\left(2^{-j\left(s_{1}-1 / 2\right)} \sqrt{\frac{\ln n}{n}}+2^{-j\left(s_{2}+1 / 2\right)} \sqrt{\frac{\ln n}{n}}+2^{j} \frac{\ln n}{n}\right) .
$$

- Suppose that H1, H2, H3 and (5.6) hold. Let $\alpha_{\tau, k}$ and $c_{\tau, k}$ be given by (3.1), and $\hat{\alpha}_{\tau, k}$ and $\hat{c}_{\tau, k}$ be given by (3.4). Then there exists a constant $C>0$ such that

$$
\mathbb{E}\left(\left|\hat{\alpha}_{\tau, k} \hat{c}_{\tau, k}-\alpha_{\tau, k} c_{\tau, k}\right|\right) \leq C \sqrt{\frac{\ln n}{n}} .
$$

Theorem 5.3 investigates the upper bound of the MAE of our estimator in the $\alpha$-mixing case.

Theorem 5.3 Consider the nonparametric regression model, defined by (1.1). Suppose that H1, H2, H3, H4 $\left(s_{1}\right)$ with $s_{1}>3 / 2, \mathbf{H 5}\left(s_{2}\right)$ with $s_{2}>1 / 2$ and (5.6) hold. Let $\delta$ be given by (1.2) and $\hat{\delta}$ be given by (3.3) with $j_{0}$ such that $(n / \ln n)^{1 / 4}<2^{j_{0}+1} \leq 2(n / \ln n)^{1 / 4}$. Then there exists a constant $C>0$ such that

$$
\mathbb{E}(|\hat{\delta}-\delta|) \leq C \sqrt{\frac{\ln n}{n}} .
$$

\section{Simulation results}

In this section, we present a simulation study designed to illustrate the finite-sample performance of the proposed wavelet density-weighted average derivative estimator $\hat{\delta}(3.3)$. We consider the nonparametric regression model (1.1) whith i.i.d. $X_{1}, \ldots, X_{n}$ having a common unknown density function $g$ and the error $\left(\xi_{t}\right)_{t \in \mathbb{Z}}$ is an autoregressive process of order one $(\operatorname{AR}(1))$ given by

$$
\xi_{i}=\alpha \xi_{i-1}+\epsilon_{i}
$$

where $\left(\epsilon_{t}\right)_{t \in \mathbb{Z}}$ is a sequence of i.i.d. random variables having the normal distribution $N\left(0, \sigma_{\epsilon}^{2}\right)$. Note that $Y_{1}, \ldots, Y_{n}$ are dependent, $\left(\xi_{t}\right)_{t \in \mathbb{Z}}$ is strictly stationary and strongly mixing for $|\alpha|<1$, (see, e.g., Doukhan (1994) and Carrasco and Chen (2002)) and the variance of $\xi_{1}$ is $\sigma_{\xi}^{2}=\sigma_{\epsilon}^{2} /\left(1-\alpha^{2}\right)$. We aim to estimate $\delta(1.2)$ from $\left(X_{i}, Y_{i}\right)$ 's data generated according to (1.1). The performance of the proposed method was studied for two sets of designs distribution for $X_{i}$, a $\operatorname{Beta}(2,2)$ (i.e., $g_{1}(x)=6 x(1-x)$ ) and a $\operatorname{Beta}(3,3)$ (i.e., $\left.g_{2}(x)=30 x^{2}(1-x)^{2}\right)$ with three test regression functions (see Figure 1 ). They are defined by 


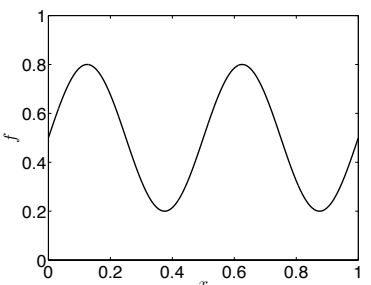

(a)

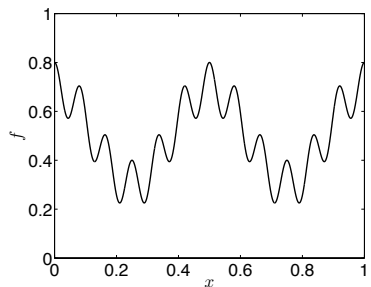

(b)

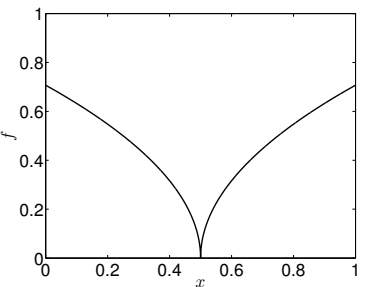

(c)

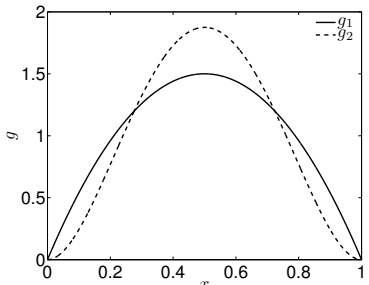

(d)

Figure 1: Theoretical regression functions (a): $f_{1}$. (b): $f_{2}$. (c): $f_{3}$. Design densities (d): $g_{1}$ and $g_{2}$.

(a) Sine:

$$
f_{1}(x)=0.5+0.3 \sin (4 \pi x) .
$$

(b) Wave (see Marron et al. (1998)):

$$
f_{2}(x)=0.5+0.2 \cos (4 \pi x)+0.1 \cos (24 \pi x) .
$$

(a) Cusp:

$$
f_{3}(x)=\sqrt{|x-0.5|}
$$

The primary level is $\tau=0$, and the Symmlet wavelet with 6 vanishing moments were used throughout all experiments. Here, $j_{0}=\log _{2}(n) / 2$, thus we keep only the $2^{j_{0}}$ wavelet coefficients to perform the reconstruction. We conduct $N=100$ Monte Carlo replications for each experiment on samples of size $n=256,512,1024$ and 2048. The MAE performance is computed as $\operatorname{MAE}(\hat{\delta})=N^{-1} \sum_{i=1}^{N}\left|\hat{\delta}_{i}-\delta_{i}\right|$. All simulations were carried out using Matlab.

It is also of interest to make comparisons with the popular kernel estimator developed by Powell et al. (1989) and the proposed estimator. More precisely, we consider the kernel estimator defined as follow

$$
\hat{\delta}^{K}=-\frac{2}{n} \sum_{i=1}^{n} Y_{i} \hat{g}_{i}^{\prime}\left(X_{i}\right),
$$

where

$$
\hat{g}_{i}^{\prime}(x)=\frac{1}{(n-1) h^{2}} \sum_{\substack{j=1 \\ j \neq i}}^{n} K^{\prime}\left(\frac{x-X_{j}}{h}\right),
$$

$h$ is the bandwidth and $K^{\prime}$ denotes the derivative of a kernel function $K$. This estimator only makes sense if $K^{\prime}$ exists and is non-zero. Since the Gaussian kernel has derivatives 
of all orders this is a common choice for density derivative estimation. Even if no theory exists in this dependent context, for the sake of simplicity, the Silverman rule-of-thumb (rot) is used to select the bandwidth. Indeed, this rule may also be applied to density derivative estimation and, since we use second order Gaussian kernel, the rot bandwidth is $h_{\text {rot }}=0.97 \hat{\sigma} n^{-1 / 7}$, where $\hat{\sigma}$ is the sample standard deviation (see, e.g., Hansen (2009)).

We study the influence of the noise level (i.e., the variance of the $\operatorname{AR}(1)$-process $\sigma_{\xi}^{2}$, ranging from "low noise" with $\sigma_{\epsilon}=0.02$, and $\alpha=0.05$, thus $\sigma_{\xi}=0.02$ through "medium noise" with $\sigma_{\epsilon}=0.06$, and $\alpha=0.6$, thus $\sigma_{\xi}=0.075$ to "high noise" with $\sigma_{\epsilon}=0.1$, and $\alpha=0.7$, thus $\sigma_{\xi}=0.14$ ) on the estimators.

Table 1 reports the mean of the MAE over 100 replications, calculated across the sampled times for each realization. As expected, increasing the variance of the AR(1)-process increases the MAE and the MAE is decreasing as the sample size increases. Our wavelet estimator is slightly better than the Kernel one in almost all cases but none of them clearly outperforms the others for all tests functions, level of noise and all sample sizes.

\section{Conclusion}

In this paper we introduce a new density-weighted average derivative estimator using wavelet methods. We evaluate its theoretical performances under various dependence assumptions on the observations. In particular, Theorems 5.1, 5.2 and 5.3 imply the consistency of our estimator (3.3), i.e. $\lim _{n \rightarrow \infty} \hat{\delta} \stackrel{p}{=} \delta$, for the considered dependence structures. This illustrates the flexibility of our approach. Our results could be useful to econometricians and statisticians working with density-weighted average derivative estimation, as a simple theory using dependent observations has been absent in this literature until now.

\section{Proofs}

\subsection{On the construction of $\hat{\delta}$}

\section{Proof of Proposition 3.1}

Using $\operatorname{supp}\left(X_{1}\right)=[0,1], g(0)=g(1)=0$ and an integration by part, we obtain

$$
\delta=\left[g^{2}(x) f(x)\right]_{0}^{1}-2 \int_{0}^{1} f(x) g(x) g^{\prime}(x) d x=-2 \int_{0}^{1} f(x) g(x) g^{\prime}(x) d x .
$$

Since $f g \in \mathbb{L}^{2}([0,1])$ and $g^{\prime} \in \mathbb{L}^{2}([0,1])$, we can expand $f g$ on $\mathcal{B}$ as $(2.1)$ :

$$
f(x) g(x)=\sum_{k \in \Lambda_{\tau}} \alpha_{\tau, k} \phi_{\tau, k}(x)+\sum_{j=\tau}^{\infty} \sum_{k \in \Lambda_{j}} \beta_{j, k} \psi_{j, k}(x),
$$


Table 1: $100 \times$ mean MAE values of estimator (3.3), from 100 replications of the model (1.1) of sample sizes 256, 512, 1024 and 2048.

\begin{tabular}{|c|c|c|c|c|c|c|c|c|}
\hline & \multicolumn{8}{|c|}{$\sigma_{\xi}=0.02$} \\
\hline & \multicolumn{4}{|c|}{$g_{1}$} & \multicolumn{4}{|c|}{$g_{2}$} \\
\hline$n$ & 256 & 512 & 1024 & 2048 & 256 & 512 & 1024 & 2048 \\
\hline $\operatorname{MAE}\left(\hat{\delta}_{f_{1}}\right)$ & 16.995 & 10.874 & 7.368 & 5.463 & 21.306 & 14.124 & 10.853 & 7.767 \\
\hline $\operatorname{MAE}\left(\hat{\delta}_{f_{1}}^{K}\right)$ & 26.595 & 28.040 & 25.159 & 22.465 & 46.443 & 50.442 & 54.003 & 57.082 \\
\hline $\operatorname{MAE}\left(\hat{\delta}_{f_{2}}\right)$ & 13.161 & 9.299 & 6.527 & 4.555 & 15.404 & 11.626 & 8.088 & 5.852 \\
\hline $\operatorname{MAE}\left(\hat{\delta}_{f_{2}}^{K}\right)$ & 13.401 & 9.184 & 6.030 & 4.949 & 18.633 & 13.262 & 10.741 & 7.169 \\
\hline $\operatorname{MAE}\left(\hat{\delta}_{f_{3}}\right)$ & 16.049 & 10.838 & 7.574 & 5.373 & 17.800 & 12.659 & 10.017 & 6.695 \\
\hline \multirow[t]{2}{*}{$\operatorname{MAE}\left(\hat{\delta}_{f_{3}}^{K}\right)$} & 12.389 & 8.816 & 6.065 & 4.710 & 15.960 & 11.290 & 9.090 & 6.309 \\
\hline & \multicolumn{8}{|c|}{$\sigma_{\xi}=0.075$} \\
\hline $\operatorname{MAE}\left(\hat{\delta}_{f_{1}}\right)$ & 16.499 & 10.857 & 6.555 & 6.369 & 32.286 & 34.796 & 32.016 & 34.532 \\
\hline $\operatorname{MAE}\left(\hat{\delta}_{f_{1}}^{K}\right)$ & 28.144 & 24.984 & 24.802 & 22.961 & 49.598 & 49.585 & 49.357 & 51.668 \\
\hline $\operatorname{MAE}\left(\hat{\delta}_{f_{2}}\right)$ & 12.637 & 9.448 & 5.858 & 5.095 & 15.978 & 14.015 & 8.961 & 5.729 \\
\hline $\operatorname{MAE}\left(\hat{\delta}_{f_{2}}^{K}\right)$ & 13.230 & 9.089 & 6.632 & 5.864 & 12.902 & 10.682 & 6.936 & 4.517 \\
\hline $\operatorname{MAE}\left(\hat{\delta}_{f_{3}}\right)$ & 15.758 & 11.163 & 6.918 & 6.425 & 18.598 & 16.780 & 9.879 & 7.175 \\
\hline \multirow[t]{2}{*}{$\operatorname{MAE}\left(\hat{\delta}_{f_{3}}^{K}\right)$} & 11.834 & 8.746 & 6.209 & 5.363 & 11.062 & 10.098 & 6.652 & 4.484 \\
\hline & \multicolumn{8}{|c|}{$\sigma_{\xi}=0.14$} \\
\hline $\operatorname{MAE}\left(\hat{\delta}_{f_{1}}\right)$ & 14.874 & 9.934 & 7.500 & 5.044 & 34.457 & 32.840 & 33.062 & 33.222 \\
\hline $\operatorname{MAE}\left(\hat{\delta}_{f_{1}}^{K}\right)$ & 26.266 & 25.873 & 24.093 & 20.847 & 45.886 & 51.442 & 50.582 & 52.013 \\
\hline $\operatorname{MAE}\left(\hat{\delta}_{f_{2}}\right)$ & 12.093 & 8.196 & 6.759 & 4.377 & 18.663 & 12.750 & 9.186 & 6.622 \\
\hline $\operatorname{MAE}\left(\hat{\delta}_{f_{2}}^{K}\right)$ & 12.594 & 9.668 & 8.074 & 5.340 & 14.944 & 9.628 & 7.558 & 4.862 \\
\hline $\operatorname{MAE}\left(\hat{\delta}_{f_{3}}\right)$ & 14.385 & 9.923 & 8.390 & 5.215 & 21.728 & 15.784 & 12.041 & 7.256 \\
\hline $\operatorname{MAE}\left(\hat{\delta}_{f_{3}}^{K}\right)$ & 11.807 & 9.246 & 7.335 & 4.650 & 13.235 & 8.812 & 7.480 & 4.931 \\
\hline
\end{tabular}

where $\alpha_{\tau, k}$ and $\beta_{j, k}$ are (3.1), and

$$
g^{\prime}(x)=\sum_{k \in \Lambda_{\tau}} c_{\tau, k} \phi_{\tau, k}(x)+\sum_{j=\tau}^{\infty} \sum_{k \in \Lambda_{j}} d_{j, k} \psi_{j, k}(x),
$$


where $c_{\tau, k}$ and $d_{j, k}$ are (3.2). Observing that the integral term in (7.1) is the scalar product of $f g$ and $g^{\prime}$, the orthonormality of $\mathcal{B}$ on $\mathbb{L}^{2}([0,1])$ yields

$$
\delta=-2 \int_{0}^{1} f(x) g(x) g^{\prime}(x) d x=-2\left(\sum_{k \in \Lambda_{\tau}} \alpha_{\tau, k} c_{\tau, k}+\sum_{j=\tau}^{\infty} \sum_{k \in \Lambda_{j}} \beta_{j, k} d_{j, k}\right) .
$$

Proposition 3.1 is proved.

\section{Proof of Proposition 3.2}

- Since $\left(Y_{1}, X_{1}\right), \ldots,\left(Y_{n}, X_{n}\right)$ are identically distributed, $\xi_{i}$ and $X_{i}$ are independent for any $i \in\{1, \ldots, n\}$, and $\mathbb{E}\left(\xi_{1}\right)=0$, we have

$$
\mathbb{E}\left(\hat{\beta}_{j, k}\right)=\mathbb{E}\left(Y_{1} \psi_{j, k}\left(X_{1}\right)\right)=\mathbb{E}\left(f\left(X_{1}\right) \psi_{j, k}\left(X_{1}\right)\right)=\int_{0}^{1} f(x) g(x) \psi_{j, k}(x) d x=\beta_{j, k} .
$$

Similarly, we prove that $\mathbb{E}\left(\hat{\alpha}_{\tau, k}\right)=\alpha_{\tau, k}$.

- Using the identical distribution of $X_{1}, \ldots, X_{n}, \mathbb{E}\left(\xi_{1}\right)=0$, an integration by parts and $g(0)=g(1)=0$, we obtain

$$
\begin{aligned}
\mathbb{E}\left(\hat{d}_{j, k}\right) & =-\mathbb{E}\left(\left(\psi_{j, k}\right)^{\prime}\left(X_{1}\right)\right)=-\int_{0}^{1} g(x)\left(\psi_{j, k}\right)^{\prime}(x) d x \\
& =-\left(\left[g(x) \psi_{j, k}(x)\right]_{0}^{1}-\int_{0}^{1} g^{\prime}(x) \psi_{j, k}(x) d x\right)=\int_{0}^{1} g^{\prime}(x) \psi_{j, k}(x) d x=d_{j, k} .
\end{aligned}
$$

Similarly, we prove that $\mathbb{E}\left(\hat{c}_{\tau, k}\right)=c_{\tau, k}$.

This ends the proof of Proposition 3.2.

\subsection{Proof of the main results}

\subsubsection{The independent case}

In the sequel, we assume that $\left(X_{1}, Y_{1}\right), \cdots,\left(X_{n}, Y_{n}\right)$ are independent. To bound the fouth cental moment of the estimators, defined by (3.4) and (3.5), we use the following version of the Rosenthal inequality (see Rosenthal (1970)). 
Lemma 7.1 Let $n$ be a positive integer, $p \geq 2$ and $U_{1}, \ldots, U_{n}$ be $n$ zero mean independent random variables such that $\sup _{i \in\{1, \ldots, n\}} \mathbb{E}\left(\left|U_{i}\right|^{p}\right)<\infty$. Then there exists a constant $C>0$ such that

$$
\mathbb{E}\left(\left|\sum_{i=1}^{n} U_{i}\right|^{p}\right) \leq C\left(\sum_{i=1}^{n} \mathbb{E}\left(\left|U_{i}\right|^{p}\right)+\left(\sum_{i=1}^{n} \mathbb{E}\left(U_{i}^{2}\right)\right)^{p / 2}\right) .
$$

\section{Proof of Proposition 5.1}

- Observe that

$$
\mathbb{E}\left(\left(\hat{\beta}_{j, k}-\beta_{j, k}\right)^{4}\right)=\frac{1}{n^{4}} \mathbb{E}\left(\left(\sum_{i=1}^{n}\left(Y_{i} \psi_{j, k}\left(X_{i}\right)-\beta_{j, k}\right)\right)^{4}\right) .
$$

Set

$$
U_{i}=Y_{i} \psi_{j, k}\left(X_{i}\right)-\beta_{j, k}, \quad i \in\{1, \ldots, n\} .
$$

Since $\left(X_{1}, Y_{1}\right), \ldots,\left(X_{n}, Y_{n}\right)$ are i.i.d., we get that $U_{1}, \ldots, U_{n}$ are also i.i.d.. Moreover, from Proposition 3.2, we have $\mathbb{E}\left(U_{1}\right)=0$. Thus, Lemma 7.1 (with $p=4$ ) yields

$$
\mathbb{E}\left(\left(\hat{\beta}_{j, k}-\beta_{j, k}\right)^{4}\right) \leq C \frac{1}{n^{4}}\left(n \mathbb{E}\left(U_{1}^{4}\right)+n^{2}\left(\mathbb{E}\left(U_{1}^{2}\right)\right)^{2}\right) .
$$

Using H1, H2, the Hölder inequality, H3, the independence between $\xi_{1}$ and $X_{1}$, $\mathbb{E}\left(\xi_{1}^{4}\right)<\infty$, applying the change of variables $y=2^{j} x-k$, and using the fact that $\psi$ is compactly supported, we have for any $u \in\{2,4\}$,

$$
\begin{aligned}
\mathbb{E}\left(U_{1}^{u}\right) & \leq C \mathbb{E}\left(\left(Y_{1} \psi_{j, k}\left(X_{1}\right)\right)^{u}\right) \leq C\left(C_{1}^{u}+\mathbb{E}\left(\xi_{1}^{u}\right)\right) \mathbb{E}\left(\left(\psi_{j, k}\left(X_{1}\right)\right)^{u}\right) \\
& =C \int_{0}^{1}\left(\psi_{j, k}(x)\right)^{u} g(x) d x \leq C \int_{0}^{1}\left(\psi_{j, k}(x)\right)^{u} d x \\
& =C 2^{j(u-2) / 2} \int_{0}^{1}(\psi(x))^{u} d x \leq C 2^{j(u-2) / 2} .
\end{aligned}
$$

Therefore, since $2^{j} \leq n$, we obtain

$$
\mathbb{E}\left(\left(\hat{\beta}_{j, k}-\beta_{j, k}\right)^{4}\right) \leq C\left(\frac{1}{n^{3}} 2^{j}+\frac{1}{n^{2}}\right) \leq C \frac{1}{n^{2}} .
$$

- We have

$$
\mathbb{E}\left(\left(\hat{d}_{j, k}-d_{j, k}\right)^{4}\right)=\frac{1}{n^{4}} \mathbb{E}\left(\left(\sum_{i=1}^{n}\left(\left(\psi_{j, k}\right)^{\prime}\left(X_{i}\right)-d_{j, k}\right)\right)^{4}\right)
$$


Now, set

$$
U_{i}=\left(\psi_{j, k}\right)^{\prime}\left(X_{i}\right)-d_{j, k}, \quad i \in\{1, \ldots, n\} .
$$

Since $X_{1}, \ldots, X_{n}$ are i.i.d., it is clear that $U_{1}, \ldots, U_{n}$ are also i.i.d.. Moreover, by Propostion 3.2, we have $\mathbb{E}\left(U_{1}\right)=0$. Hence, Lemma 7.1 (with $p=4$ ) yields

$$
\mathbb{E}\left(\left(\hat{d}_{j, k}-d_{j, k}\right)^{4}\right) \leq C \frac{1}{n^{4}}\left(n \mathbb{E}\left(U_{1}^{4}\right)+n^{2}\left(\mathbb{E}\left(U_{1}^{2}\right)\right)^{2}\right) .
$$

Using H2, the Hölder inequality, H3, $\left(\psi_{j, k}\right)^{\prime}(x)=2^{3 j / 2} \psi^{\prime}\left(2^{j} x-k\right)$, applying the change of variables $y=2^{j} x-k$, and using the fact that $\psi$ is compactly supported and $\mathcal{C}^{1}$, we have for any $u \in\{2,4\}$,

$$
\begin{aligned}
\mathbb{E}\left(U_{1}^{u}\right) & \leq C \mathbb{E}\left(\left(\left(\psi_{j, k}\right)^{\prime}\left(X_{1}\right)\right)^{u}\right)=C \int_{0}^{1}\left(\left(\psi_{j, k}\right)^{\prime}(x)\right)^{u} g(x) d x \leq C \int_{0}^{1}\left(\left(\psi_{j, k}\right)^{\prime}(x)\right)^{u} d x \\
& =C 2^{j(3 u-2) / 2} \int_{0}^{1}\left(\psi^{\prime}(x)\right)^{u} d x \leq C 2^{j(3 u-2) / 2}
\end{aligned}
$$

Putting these inequalities together and using $2^{j} \leq n$, we obtain

$$
\mathbb{E}\left(\left(\hat{d}_{j, k}-d_{j, k}\right)^{4}\right) \leq C\left(\frac{2^{5 j}}{n^{3}}+\frac{2^{4 j}}{n^{2}}\right) \leq C \frac{2^{4 j}}{n^{2}} .
$$

Proposition 5.1 is proved.

\section{Proof of Proposition 5.2}

- We have the following decomposition

$$
\hat{\beta}_{j, k} \hat{d}_{j, k}-\beta_{j, k} d_{j, k}=\beta_{j, k}\left(\hat{d}_{j, k}-d_{j, k}\right)+d_{j, k}\left(\hat{\beta}_{j, k}-\beta_{j, k}\right)+\left(\hat{\beta}_{j, k}-\beta_{j, k}\right)\left(\hat{d}_{j, k}-d_{j, k}\right) .
$$

Therefore

$$
\mathbb{E}\left(\left(\hat{\beta}_{j, k} \hat{d}_{j, k}-\beta_{j, k} d_{j, k}\right)^{2}\right) \leq 3\left(T_{1}+T_{2}+T_{3}\right)
$$

where

$$
T_{1}=\beta_{j, k}^{2} \mathbb{E}\left(\left(\hat{d}_{j, k}-d_{j, k}\right)^{2}\right), \quad T_{2}=d_{j, k}^{2} \mathbb{E}\left(\left(\hat{\beta}_{j, k}-\beta_{j, k}\right)^{2}\right)
$$

and

$$
T_{3}=\mathbb{E}\left(\left(\hat{\beta}_{j, k}-\beta_{j, k}\right)^{2}\left(\hat{d}_{j, k}-d_{j, k}\right)^{2}\right)
$$


Upper bound for $T_{1}$. It follows from the Cauchy-Schwarz inequality, the second point in Proposition 5.1 and $\mathbf{H} \mathbf{4}\left(s_{1}\right)$ that

$$
T_{1} \leq C_{4}^{2} 2^{-2 j\left(s_{1}+1 / 2\right)} \sqrt{\mathbb{E}\left(\left(\hat{d}_{j, k}-d_{j, k}\right)^{4}\right)} \leq C 2^{-2 j\left(s_{1}+1 / 2\right)} \frac{2^{2 j}}{n}=C \frac{2^{-j\left(2 s_{1}-1\right)}}{n} .
$$

Upper bound for $T_{2}$. By the Cauchy-Schwarz inequality, the first point in Proposition 5.1 and $\mathbf{H 5}\left(s_{2}\right)$, we obtain

$$
T_{2} \leq C_{5}^{2} 2^{-2 j\left(s_{2}+1 / 2\right)} \sqrt{\mathbb{E}\left(\left(\hat{\beta}_{j, k}-\beta_{j, k}\right)^{4}\right)} \leq C \frac{2^{-j\left(2 s_{2}+1\right)}}{n} .
$$

Upper bound for $T_{3}$. The Cauchy-Schwarz inequality and Proposition 5.1 yield

$$
T_{3} \leq \sqrt{\left(\mathbb{E}\left(\left(\hat{\beta}_{j, k}-\beta_{j, k}\right)^{4}\right) \mathbb{E}\left(\left(\hat{d}_{j, k}-d_{j, k}\right)^{4}\right)\right.} \leq C \sqrt{\frac{1}{n^{2}} \frac{2^{4 j}}{n^{2}}}=C \frac{2^{2 j}}{n^{2}}
$$

Combining the inequalities above, we obtain

$$
\mathbb{E}\left(\left(\hat{\beta}_{j, k} \hat{d}_{j, k}-\beta_{j, k} d_{j, k}\right)^{2}\right) \leq C\left(\frac{2^{-j\left(2 s_{1}-1\right)}}{n}+\frac{2^{-j\left(2 s_{2}+1\right)}}{n}+\frac{2^{2 j}}{n^{2}}\right) .
$$

- The proof of the second point is identical to the first one but with the bounds $\left|\alpha_{\tau, k}\right| \leq$ $C$ and $\left|c_{\tau, k}\right| \leq C$ thanks to $\mathbf{H 2}$ and $\mathbf{H 3}$.

This ends the proof of Proposition 5.2.

The following Lemma will be very usefull for the proof of Theorem 5.1. It is a consequence of the Cauchy-Schwarz inequality.

Lemma 7.2 Let $n$ be a positive integer and $U_{1}, \ldots, U_{n}$ be $n$ random variables such that $\sup _{i \in\{1, \ldots, n\}} \mathbb{E}\left(U_{i}^{2}\right)<\infty$. Then

$$
\mathbb{E}\left(\left(\sum_{i=1}^{n} U_{i}\right)^{2}\right) \leq\left(\sum_{i=1}^{n} \sqrt{\mathbb{E}\left(U_{i}^{2}\right)}\right)^{2}
$$

\section{Proof of Theorem 5.1}

It follows from Proposition 3.1 that

$$
\begin{aligned}
\hat{\delta}-\delta & =-2 \sum_{k \in \Lambda_{\tau}}\left(\hat{\alpha}_{\tau, k} \hat{c}_{\tau, k}-\alpha_{\tau, k} c_{\tau, k}\right)-2 \sum_{j=\tau}^{j_{0}} \sum_{k \in \Lambda_{j}}\left(\hat{\beta}_{j, k} \hat{d}_{j, k}-\beta_{j, k} d_{j, k}\right) \\
& +2 \sum_{j=j_{0}+1}^{\infty} \sum_{k \in \Lambda_{j}} \beta_{j, k} d_{j, k} .
\end{aligned}
$$


Therefore

$$
\mathbb{E}\left((\hat{\delta}-\delta)^{2}\right) \leq 12\left(W_{1}+W_{2}+W_{3}\right)
$$

where

$$
\begin{array}{r}
W_{1}=\mathbb{E}\left(\left(\sum_{k \in \Lambda_{\tau}}\left(\hat{\alpha}_{\tau, k} \hat{c}_{\tau, k}-\alpha_{\tau, k} c_{\tau, k}\right)\right)^{2}\right), \\
W_{2}=\mathbb{E}\left(\left(\sum_{j=\tau}^{j_{0}} \sum_{k \in \Lambda_{j}}\left(\hat{\beta}_{j, k} \hat{d}_{j, k}-\beta_{j, k} d_{j, k}\right)\right)^{2}\right)
\end{array}
$$

and

$$
W_{3}=\left(\sum_{j=j_{0}+1}^{\infty} \sum_{k \in \Lambda_{j}} \beta_{j, k} d_{j, k}\right)^{2} .
$$

Let us now bound $W_{1}$ and $W_{2}$ in turn.

Upper bound for $W_{1}$. Owing to Lemma 7.2, the second point of Proposition 5.2 and $\operatorname{Card}\left(\Lambda_{\tau}\right)=2^{\tau}$, we obtain

$$
W_{1} \leq\left(\sum_{k \in \Lambda_{\tau}} \sqrt{\mathbb{E}\left(\left(\hat{\alpha}_{\tau, k} \hat{c}_{\tau, k}-\alpha_{\tau, k} c_{\tau, k}\right)^{2}\right)}\right)^{2} \leq C \frac{1}{n} .
$$

Upper bound for $W_{2}$. It follows from Lemma 7.2, the first point of Proposition 5.2, $\operatorname{Card}\left(\Lambda_{j}\right)=2^{j}$, the elementary inequality: $\sqrt{a+b+c} \leq \sqrt{a}+\sqrt{b}+\sqrt{c}, s_{1}>3 / 2, s_{2}>1 / 2$ and $2^{j_{0}} \leq n^{1 / 4}$ that

$$
\begin{aligned}
W_{2} & \leq\left(\sum_{j=\tau}^{j_{0}} \sum_{k \in \Lambda_{j}} \sqrt{\mathbb{E}\left(\left(\hat{\beta}_{j, k} \hat{d}_{j, k}-\beta_{j, k} d_{j, k}\right)^{2}\right)}\right)^{2} \\
& \leq C\left(\sum_{j=\tau}^{j_{0}} 2^{j} \sqrt{\left.\frac{2^{-j\left(2 s_{1}-1\right)}}{n}+\frac{2^{-j\left(2 s_{2}+1\right)}}{n}+\frac{2^{2 j}}{n^{2}}\right)^{2}}\right. \\
& \leq C\left(\sum_{j=\tau}^{j_{0}}\left(\frac{2^{-j\left(s_{1}-3 / 2\right)}}{\sqrt{n}}+\frac{2^{-j\left(s_{2}-1 / 2\right)}}{\sqrt{n}}+\frac{2^{2 j}}{n}\right)\right)^{2} \\
& \leq C\left(\frac{1}{\sqrt{n}} \sum_{j=\tau}^{j_{0}} 2^{-j\left(s_{1}-3 / 2\right)}+\frac{1}{\sqrt{n}} \sum_{j=\tau}^{j_{0}} 2^{-j\left(s_{2}-1 / 2\right)}+\frac{1}{n} \sum_{j=\tau}^{j_{0}} 2^{2 j}\right)^{2} \\
& \leq C\left(\frac{1}{\sqrt{n}}+\frac{1}{\sqrt{n}}+\frac{2^{2 j_{0}}}{n}\right)^{2} \leq C \frac{1}{n} .
\end{aligned}
$$


Upper bound for $W_{3}$. By $\mathbf{H 4}\left(s_{1}\right)$ with $s_{1}>3 / 2$, H5 $\left(s_{2}\right)$ with $s_{2}>1 / 2$ and $2^{j_{0}+1}>n^{1 / 4}$, we have

$$
\begin{aligned}
W_{3} & \leq\left(\sum_{j=j_{0}+1}^{\infty} \sum_{k \in \Lambda_{j}}\left|\beta_{j, k} \| d_{j, k}\right|\right)^{2} \leq C\left(\sum_{j=j_{0}+1}^{\infty} 2^{j} 2^{-j\left(s_{1}+1 / 2\right)} 2^{-j\left(s_{2}+1 / 2\right)}\right)^{2} \leq C 2^{-2 j_{0}\left(s_{1}+s_{2}\right)} \\
& \leq C 2^{-4 j_{0}} \leq C \frac{1}{n} .
\end{aligned}
$$

Putting (7.4), (7.5), (7.6) and (7.7) together, we obtain

$$
\mathbb{E}\left((\hat{\delta}-\delta)^{2}\right) \leq C \frac{1}{n}
$$

This ends the proof of Theorem 5.1.

\subsubsection{The $\rho$-mixing case}

In the sequel, we assume that $\left(X_{1}, Y_{1}\right), \ldots,\left(X_{n}, Y_{n}\right)$ coming from a $\rho$-mixing strictly stationary process $\left(X_{t}, Y_{t}\right)_{t \in \mathbb{Z}}(1.1)$ (see Definition 2.1).

\section{Proof of Proposition 5.3}

- From Proposition 3.2 , we have $\mathbb{E}\left(\hat{\beta}_{j, k}\right)=\beta_{j, k}$. It follows that

$$
\mathbb{E}\left(\left(\hat{\beta}_{j, k}-\beta_{j, k}\right)^{2}\right)=\frac{1}{n^{2}} \mathbb{V}\left(\sum_{i=1}^{n} Y_{i} \psi_{j, k}\left(X_{i}\right)\right)=S_{1}+S_{2},
$$

where

$$
S_{1}=\frac{1}{n} \mathbb{V}\left(Y_{1} \psi_{j, k}\left(X_{1}\right)\right), \quad S_{2}=\frac{2}{n^{2}} \sum_{v=2}^{n} \sum_{\ell=1}^{v-1} \operatorname{Cov}\left(Y_{v} \psi_{j, k}\left(X_{v}\right), Y_{\ell} \psi_{j, k}\left(X_{\ell}\right)\right) .
$$

Upper bound for $S_{1}$. It follows from (7.2) with $u=2$ that

$$
S_{1} \leq \frac{1}{n} \mathbb{E}\left(\left(Y_{1} \psi_{j, k}\left(X_{1}\right)\right)^{2}\right) \leq C \frac{1}{n} .
$$

Upper bound for $S_{2}$. The stationarity of $\left(X_{t}, Y_{t}\right)_{t \in \mathbb{Z}}$ implies that

$$
\begin{aligned}
S_{2} & =\frac{2}{n^{2}} \sum_{m=1}^{n-1}(n-m) \operatorname{Cov}\left(Y_{m+1} \psi_{j, k}\left(X_{m+1}\right), Y_{1} \psi_{j, k}\left(X_{1}\right)\right) \\
& \leq \frac{2}{n} \sum_{m=1}^{n-1}\left|\operatorname{Cov}\left(Y_{m+1} \psi_{j, k}\left(X_{m+1}\right), Y_{1} \psi_{j, k}\left(X_{1}\right)\right)\right|
\end{aligned}
$$


A standard covariance inequality for $\rho$-mixing gives

$$
\left|\operatorname{Cov}\left(Y_{m+1} \psi_{j, k}\left(X_{m+1}\right), Y_{1} \psi_{j, k}\left(X_{1}\right)\right)\right| \leq \mathbb{E}\left(\left(Y_{1} \psi_{j, k}\left(X_{1}\right)\right)^{2}\right) \rho_{m}
$$

(see, for instance, (Zhengyan and Lu, 1996, Lemma 1.2.7.)).

Equation (7.2) with $u=2$ yields

$$
\mathbb{E}\left(\left(Y_{1} \psi_{j, k}\left(X_{1}\right)\right)^{2}\right) \leq C .
$$

Therefore, using (5.5),

$$
S_{2} \leq C \frac{1}{n} \sum_{m=1}^{n-1} \rho_{m} \leq C \frac{1}{n} \sum_{m=1}^{\infty} \rho_{m} \leq C \frac{1}{n} .
$$

Combining the inequalities above, we obtain

$$
\mathbb{E}\left(\left(\hat{\beta}_{j, k}-\beta_{j, k}\right)^{2}\right) \leq C \frac{1}{n} .
$$

- Proceeding as for the first point but with $\left(\psi_{j, k}\right)^{\prime}\left(X_{i}\right)$ instead of $Y_{i} \psi_{j, k}\left(X_{i}\right)$ and $(7.3)$ instead of (7.2).

Proposition 5.3 is proved.

\section{Proof of Proposition 5.4}

- We have the following decomposition

$$
\hat{\beta}_{j, k} \hat{d}_{j, k}-\beta_{j, k} d_{j, k}=\beta_{j, k}\left(\hat{d}_{j, k}-d_{j, k}\right)+d_{j, k}\left(\hat{\beta}_{j, k}-\beta_{j, k}\right)+\left(\hat{\beta}_{j, k}-\beta_{j, k}\right)\left(\hat{d}_{j, k}-d_{j, k}\right) .
$$

Therefore

$$
\mathbb{E}\left(\left|\hat{\beta}_{j, k} \hat{d}_{j, k}-\beta_{j, k} d_{j, k}\right|\right) \leq T_{1}+T_{2}+T_{3},
$$

where

$$
T_{1}=\left|\beta_{j, k}\right| \mathbb{E}\left(\left|\hat{d}_{j, k}-d_{j, k}\right|\right), \quad T_{2}=\left|d_{j, k}\right| \mathbb{E}\left(\left|\hat{\beta}_{j, k}-\beta_{j, k}\right|\right)
$$

and

$$
T_{3}=\mathbb{E}\left(\left|\left(\hat{\beta}_{j, k}-\beta_{j, k}\right)\left(\hat{d}_{j, k}-d_{j, k}\right)\right|\right) .
$$

Upper bound for $T_{1}$. Using the Cauchy-Schwarz inequality, the second point in Proposition 5.3 and $\mathbf{H} 4\left(s_{1}\right)$, we obtain

$$
T_{1} \leq C_{4} 2^{-j\left(s_{1}+1 / 2\right)} \sqrt{\mathbb{E}\left(\left(\hat{d}_{j, k}-d_{j, k}\right)^{2}\right)} \leq C 2^{-j\left(s_{1}+1 / 2\right)} \frac{2^{j}}{\sqrt{n}}=C \frac{2^{-j\left(s_{1}-1 / 2\right)}}{\sqrt{n}} .
$$


Upper bound for $T_{2}$. By the Cauchy-Schwarz inequality, the first point in Proposition 5.3 and $\mathbf{H 5}\left(s_{2}\right)$, we obtain

$$
T_{2} \leq C_{5} 2^{-j\left(s_{2}+1 / 2\right)} \sqrt{\mathbb{E}\left(\left(\hat{\beta}_{j, k}-\beta_{j, k}\right)^{2}\right)} \leq C \frac{2^{-j\left(s_{2}+1 / 2\right)}}{\sqrt{n}} .
$$

Upper bound for $T_{3}$. The Cauchy-Schwarz inequality and Proposition 5.3 yield

$$
T_{3} \leq \sqrt{\left(\mathbb{E}\left(\left(\hat{\beta}_{j, k}-\beta_{j, k}\right)^{2}\right) \mathbb{E}\left(\left(\hat{d}_{j, k}-d_{j, k}\right)^{2}\right)\right.} \leq C \sqrt{\frac{1}{n} \frac{2^{2 j}}{n}}=C \frac{2^{j}}{n} .
$$

The above inequalities imply that

$$
\mathbb{E}\left(\left|\hat{\beta}_{j, k} \hat{d}_{j, k}-\beta_{j, k} d_{j, k}\right|\right) \leq C\left(\frac{2^{-j\left(s_{1}-1 / 2\right)}}{\sqrt{n}}+\frac{2^{-j\left(s_{2}+1 / 2\right)}}{\sqrt{n}}+\frac{2^{j}}{n}\right) .
$$

- The proof of the second point is identical to the first one but with the bounds $\left|\alpha_{\tau, k}\right| \leq$ $C$ and $\left|c_{\tau, k}\right| \leq C$ thanks to $\mathbf{H 2}$ and $\mathbf{H 3}$.

This ends the proof of Proposition 5.4.

\section{Proof of Theorem 5.2}

Using Proposition 3.1, we have

$$
\begin{aligned}
\hat{\delta}-\delta & =-2 \sum_{k \in \Lambda_{\tau}}\left(\hat{\alpha}_{\tau, k} \hat{c}_{\tau, k}-\alpha_{\tau, k} c_{\tau, k}\right)-2 \sum_{j=\tau}^{j_{0}} \sum_{k \in \Lambda_{j}}\left(\hat{\beta}_{j, k} \hat{d}_{j, k}-\beta_{j, k} d_{j, k}\right) \\
& +2 \sum_{j=j_{0}+1}^{\infty} \sum_{k \in \Lambda_{j}} \beta_{j, k} d_{j, k} .
\end{aligned}
$$

Therefore

$$
\mathbb{E}(|\hat{\delta}-\delta|) \leq W_{1}+W_{2}+W_{3}
$$

where

$$
W_{1}=\sum_{k \in \Lambda_{\tau}} \mathbb{E}\left(\left|\hat{\alpha}_{\tau, k} \hat{c}_{\tau, k}-\alpha_{\tau, k} c_{\tau, k}\right|\right), \quad W_{2}=\sum_{j=\tau}^{j_{0}} \sum_{k \in \Lambda_{j}} \mathbb{E}\left(\left|\hat{\beta}_{j, k} \hat{d}_{j, k}-\beta_{j, k} d_{j, k}\right|\right)
$$


and

$$
W_{3}=\sum_{j=j_{0}+1}^{\infty} \sum_{k \in \Lambda_{j}}\left|\beta_{j, k}\right|\left|d_{j, k}\right| .
$$

Upper bound for $W_{1}$. The second point of Proposition 5.4 and $\operatorname{Card}\left(\Lambda_{\tau}\right)=2^{\tau}$ give

$$
W_{1} \leq C \frac{1}{\sqrt{n}}
$$

Upper bound for $W_{2}$. It follows from the first point of Proposition 5.4, $\operatorname{Card}\left(\Lambda_{j}\right)=2^{j}$, $s_{1}>3 / 2, s_{2}>1 / 2$ and $2^{j_{0}} \leq n^{1 / 4}$ that

$$
\begin{aligned}
W_{2} & \leq C \sum_{j=\tau}^{j_{0}} 2^{j}\left(\frac{2^{-j\left(s_{1}-1 / 2\right)}}{\sqrt{n}}+\frac{2^{-j\left(s_{2}+1 / 2\right)}}{\sqrt{n}}+\frac{2^{j}}{n}\right) \\
& \leq C\left(\frac{1}{\sqrt{n}} \sum_{j=\tau}^{j_{0}} 2^{-j\left(s_{1}-3 / 2\right)}+\frac{1}{\sqrt{n}} \sum_{j=\tau}^{j_{0}} 2^{-j\left(s_{2}-1 / 2\right)}+\frac{1}{n} \sum_{j=\tau}^{j_{0}} 2^{2 j}\right) \\
& \leq C\left(\frac{1}{\sqrt{n}}+\frac{1}{\sqrt{n}}+\frac{2^{2 j_{0}}}{n}\right) \leq C \frac{1}{\sqrt{n}} .
\end{aligned}
$$

Upper bound for $W_{3}$. By $\mathbf{H 4}\left(s_{1}\right)$ with $s_{1}>3 / 2$, $\mathbf{H} 5\left(s_{2}\right)$ with $s_{2}>1 / 2$ and $2^{j_{0}+1}>n^{1 / 4}$, we have

$$
W_{3} \leq C \sum_{j=j_{0}+1}^{\infty} 2^{j} 2^{-j\left(s_{1}+1 / 2\right)} 2^{-j\left(s_{2}+1 / 2\right)} \leq C 2^{-j_{0}\left(s_{1}+s_{2}\right)} \leq C 2^{-2 j_{0}} \leq C \frac{1}{\sqrt{n}} .
$$

Putting (7.8), (7.9), (7.10) and (7.11) together, we obtain

$$
\mathbb{E}(|\hat{\delta}-\delta|) \leq C \frac{1}{\sqrt{n}}
$$

This ends the proof of Theorem 5.2.

\subsubsection{The $\alpha$-mixing case}

Recall that, here, we assume that $\left(X_{1}, Y_{1}\right), \ldots,\left(X_{n}, Y_{n}\right)$ coming from a $\alpha$-mixing strictly stationary process $\left(X_{t}, Y_{t}\right)_{t \in \mathbb{Z}}(1.1)$ (see Definition 2.2). 


\section{Proof of Proposition 5.5}

- Proposition 3.2 yields $\mathbb{E}\left(\hat{\beta}_{j, k}\right)=\beta_{j, k}$. Therefore,

$$
\mathbb{E}\left(\left(\hat{\beta}_{j, k}-\beta_{j, k}\right)^{2}\right)=\frac{1}{n^{2}} \mathbb{V}\left(\sum_{i=1}^{n} Y_{i} \psi_{j, k}\left(X_{i}\right)\right)=S_{1}+S_{2},
$$

where

$$
S_{1}=\frac{1}{n} \mathbb{V}\left(Y_{1} \psi_{j, k}\left(X_{1}\right)\right), \quad S_{2}=\frac{2}{n^{2}} \sum_{v=2}^{n} \sum_{\ell=1}^{v-1} \operatorname{Cov}\left(Y_{v} \psi_{j, k}\left(X_{v}\right), Y_{\ell} \psi_{j, k}\left(X_{\ell}\right)\right) .
$$

Upper bound for $S_{1}$. It follows from (7.2) with $u=2$ that

$$
S_{1} \leq \frac{1}{n} \mathbb{E}\left(\left(Y_{1} \psi_{j, k}\left(X_{1}\right)\right)^{2}\right) \leq C \frac{1}{n} .
$$

Upper bound for $S_{2}$. The stationarity of $\left(X_{t}, Y_{t}\right)_{t \in \mathbb{Z}}$ implies that

$$
\begin{aligned}
S_{2} & =\frac{2}{n^{2}} \sum_{m=1}^{n-1}(n-m) \operatorname{Cov}\left(Y_{m+1} \psi_{j, k}\left(X_{m+1}\right), Y_{1} \psi_{j, k}\left(X_{1}\right)\right) \\
& \leq \frac{2}{n} \sum_{m=1}^{n-1}\left|\operatorname{Cov}\left(Y_{m+1} \psi_{j, k}\left(X_{m+1}\right), Y_{1} \psi_{j, k}\left(X_{1}\right)\right)\right|
\end{aligned}
$$

Let $[c \ln n]$ be the integer part of $c \ln n$ where $c=1 / \ln b$. We have

$$
\begin{aligned}
& \sum_{m=1}^{n-1}\left|\operatorname{Cov}\left(Y_{m+1} \psi_{j, k}\left(X_{m+1}\right), Y_{1} \psi_{j, k}\left(X_{1}\right)\right)\right|= \\
& \quad \sum_{m=1}^{[c \ln n]}\left|\operatorname{Cov}\left(Y_{m+1} \psi_{j, k}\left(X_{m+1}\right), Y_{1} \psi_{j, k}\left(X_{1}\right)\right)\right| \\
& +\sum_{m=[c \ln n]+1}^{n-1}\left|\operatorname{Cov}\left(Y_{m+1} \psi_{j, k}\left(X_{m+1}\right), Y_{1} \psi_{j, k}\left(X_{1}\right)\right)\right| .
\end{aligned}
$$

On the one hand, the Cauchy-Schwarz inequality and (7.2) with $u=2$ yield

$$
\left|\operatorname{Cov}\left(Y_{m+1} \psi_{j, k}\left(X_{m+1}\right), Y_{1} \psi_{j, k}\left(X_{1}\right)\right)\right| \leq \mathbb{E}\left(\left(Y_{1} \psi_{j, k}\left(X_{1}\right)\right)^{2}\right) \leq C .
$$

Hence

$$
\sum_{m=1}^{[c \ln n]} \mid \operatorname{Cov}\left(Y_{m+1} \psi_{j, k}\left(X_{m+1}\right), Y_{1} \psi_{j, k}\left(X_{1}\right)\right) \leq C \ln n
$$


On the other hand, a standard covariance inequality for $\alpha$-mixing gives, for any $\gamma \in$ $(0,1)$,

$$
\left|\operatorname{Cov}\left(Y_{m+1} \psi_{j, k}\left(X_{m+1}\right), Y_{1} \psi_{j, k}\left(X_{1}\right)\right)\right| \leq 10 \alpha_{m}^{\gamma}\left(\mathbb{E}\left(\left|Y_{1} \psi_{j, k}\left(X_{1}\right)\right|^{2 /(1-\gamma)}\right)\right)^{1-\gamma} .
$$

(See, for instance, Davydov (1970)).

Taking $\gamma=1 / 2$ and using (5.6), again (7.2) with $u=4$ and $2^{j} \leq n$, we obtain

$$
\begin{aligned}
& \sum_{m=[c \ln n]+1}^{n-1}\left|\operatorname{Cov}\left(Y_{m+1} \psi_{j, k}\left(X_{m+1}\right), Y_{1} \psi_{j, k}\left(X_{1}\right)\right)\right| \\
& \leq C \sqrt{\mathbb{E}\left(\left(Y_{1} \psi_{j, k}\left(X_{1}\right)\right)^{4}\right)} \sum_{m=[c \ln n]+1}^{n-1} \sqrt{\alpha_{m}} \\
& \leq C 2^{j / 2} \sum_{m=[c \ln n]+1}^{\infty} b^{-m / 2} \leq C \sqrt{n} b^{-c \ln n / 2} \leq C .
\end{aligned}
$$

Hence

$$
\sum_{m=[c \ln n]+1}^{n-1}\left|\operatorname{Cov}\left(Y_{m+1} \psi_{j, k}\left(X_{m+1}\right), Y_{1} \psi_{j, k}\left(X_{1}\right)\right)\right| \leq C
$$

Then

$$
S_{2} \leq C \frac{\ln n}{n} .
$$

Combining the inequalities above, we obtain

$$
\mathbb{E}\left(\left(\hat{\beta}_{j, k}-\beta_{j, k}\right)^{2}\right) \leq C \frac{\ln n}{n} .
$$

- The proof is similar to the first point. It is enough to replace $Y_{i} \psi_{j, k}\left(X_{i}\right)$ by $\left(\psi_{j, k}\right)^{\prime}\left(X_{i}\right)$, apply (7.3) instead of (7.2) and observe that

$$
\begin{aligned}
& \sum_{m=[c \ln n]+1}^{n-1}\left|\operatorname{Cov}\left(\left(\psi_{j, k}\right)^{\prime}\left(X_{m+1}\right),\left(\psi_{j, k}\right)^{\prime}\left(X_{1}\right)\right)\right| \\
& \leq C \sqrt{\mathbb{E}\left(\left(\left(\psi_{j, k}\right)^{\prime}\left(X_{1}\right)\right)^{4}\right)} \sum_{m=[c \ln n]+1}^{n-1} \sqrt{\alpha_{m}} \\
& \leq C 2^{3 j / 2} 2^{j} \sum_{m=[c \ln n]+1}^{\infty} b^{-m / 2} \leq C 2^{2 j} \sqrt{n} b^{-c \ln n / 2} \leq C 2^{2 j} .
\end{aligned}
$$

Proposition 5.5 is proved. 


\section{Proof of Proposition 5.6}

The proof of Proposition 5.6 is identical to the one of Proposition 5.4. It is enough to use Proposition 5.5 instead of Proposition 5.3 and to replace $1 / n$ by $\ln n / n$.

\section{Proof of Theorem 5.3}

The proof of Theorem 5.3 is identical to the one of Theorem 5.2. It suffices to use Proposition 5.6 instead of Proposition 5.4 and to replace $1 / n$ by $\ln n / n$.

Acknowledgments. The author thanks two anonymous referees for their thorough and useful comments.

\section{References}

Antoniadis, A. (1997). Wavelets in statistics: a review (with discussion). Journal of the Italian Statistical Society, Series B, 6, 97-144.

Banerjee, A.N. (2007). A method of estimating the Average Derivative. Journal of Econometrics, 136,(1), 65-88.

Cattaneo, M.D., Crump, R.K. and Jansson, M. (2010). Robust Data-Driven Inference for Density-Weighted Average Derivatives. Journal of the American Statistical Association, 105, (491), 1070-1083.

Cattaneo, M.D., Crump, R.K. and Jansson, M. (2011). Small Bandwidth Asymptotics for Density-Weighted Average Derivatives. Econometric Theory, (to appear).

Carrasco, M. and Chen, X. (2002). Mixing and moment properties of various GARCH and stochastic volatility models. Econometric Theory, 18, 17-39.

Chaubey, Y.P. and Doosti, H. (2005). Wavelet based estimation of the derivatives of a density for m-dependent random variables. Journal of the Iranian Statistical Society, 4, (2), 97-105.

Chaubey, Y.P., Doosti, H. and Prakasa Rao, B.L.S. (2006). Wavelet based estimation of the derivatives of a density with associated variables. International Journal of Pure and Applied Mathematics, 27, (1), 97-106.

Cohen, A., Daubechies, I., Jawerth, B. and Vial, P. (1993). Wavelets on the interval and fast wavelet transforms. Applied and Computational Harmonic Analysis, 24, 1, 54-81. 
Coppejans, M. and Sieg, H. (2005). Kernel Estimation of Average Derivatives and Differences. Journal of Business and Economic Statistics, 23, 211-225.

Daubechies, I. (1992). Ten Lectures on Wavelets. CBMS-NSF regional conferences series in applied mathematics. SIAM, Philadelphia.

Davydov, Y. (1970). The invariance principle for stationary processes. Theor. Probab. Appl., $15,3,498-509$.

Deaton, A. and Ng, S. (1998). Parametric and Nonparametric Approaches to Price and Tax Reform. Journal of the American Statistical Association, 93, 900-909.

Doukhan, P. (1994). Mixing. Properties and Examples. Lecture Notes in Statistics 85. Springer Verlag, New York.

Fryzlewicz, P. and Subba Rao, S. (2011). Mixing properties of ARCH and time-varying ARCH processes. Bernoulli, Volume 17, Number 1 (2011), 320-346.

Härdle, W. and Stoker, T.M. (1989). Investigating smooth multiple regression by the method of average derivatives. J. Amer. Statist. Assoc., 84, 986-995.

Härdle, W., Hildenbrand, W. and Jerison, M. (1991). Empirical Evidence on the Law of Demand. Econometrica, 59, 1525-1549.

Härdle, W., Hart, J., Marron, J.S. and Tsybakov, A.B. (1992), Bandwidth Choice for Average Derivative Estimation. Journal of the American Statistical Association, 87, 417. 218-226.

Härdle, W. and Tsybakov, A.B. (1993). How Sensitive are Average Derivatives?. Journal of Econometrics, 58, 31-48.

Härdle, W., Kerkyacharian, G., Picard, D. and Tsybakov, A. (1998). Wavelet, Approximation and Statistical Applications. Lectures Notes in Statistics, New York 129, Springer Verlag.

Hansen, B. (2009), Lecture notes on nonparametrics, Lecture notes.

Juditsky, A. and Lambert-Lacroix, S. (2004), On minimax density estimation on R, Bernoulli, 10, 187-220.

Kolmogorov, A.N. and Rozanov, Yu.A. (1960). On strong mixing conditions for stationary Gaussian processes. Theor. Probab. Appl., 5, 204-208.

Lütkepohl, H. (1992). Multiple Time Series Analysis. Springer-Verlag, Heidelberg. 
Mallat, S. (2009). A wavelet tour of signal processing. Elsevier/ Academic Press, Amsterdam, third edition. The sparse way, With contributions from Gabriel Peyré.

Marron, J.S., Adak, S., Johnstone, I.M., Neumann, M.H. and Patil, P. (1998). Exact risk analysis of wavelet regression. J. Comput. Graph. Statist, 7, 278-309.

Matzkin, R. L. (2007). Nonparametric identification, in Handbook of Econometrics. Volume VIB, ed. by J. Heckman, and E. Leamer, pp. 5307-5368. Elsevier Science B. V..

Meyer, Y. (1992). Wavelets and Operators. Cambridge University Press, Cambridge.

Powell, J.L., Stock, J.H. and Stoker, T.M. (1989). Semiparametric estimation of index coefficients. Econometrica, 57, 1403-1430.

Powell, J.L. (1994). Estimation of Semiparametric Models, in Handbook of Econometrics, Volume IV, ed. by R. Engle, and D. McFadden, pp. 2443-2521. Elsevier Science B. V..

Powell, J.L. and Stoker, T.M. (1996). Optimal Bandwidth Choice For Density-Weighted Averages. Journal of Econometrics, 75, 291-316.

Prakasa Rao, B.L.S. (1995). Consistent estimation of density-weighted average derivative by orthogonal series method. Statistics and Probability Letters, 22, 205-212.

Prakasa Rao, B.L.S. (1996). Nonparametric estimation of the derivatives of a density by the method of wavelets. Bull. Inform. Cyb, 28, 91-10.

Reynaud-Bouret, P., Rivoirard, V. and Tuleau-Malot, C. (2011). Adaptive density estimation: a curse of support?. Journal of Statistical Planning and Inference, 141, 115-139.

Rosenblatt, M. (1956). A central limit theorem and a strong mixing condition. Proc. Nat. Ac. Sc. USA, 42, 43-47.

Rosenthal, H.P. (1970). On the subspaces of $\mathbb{L}_{p}(p \geq 2)$ spanned by sequences of independent random variables. Israel Journal of Mathematics, 8, 273-303.

Schafgans, M. and Zinde-Walsh, V. (2010). Smoothness adaptive average derivative estimation. Econometrics journal, 13, (1), 40-62.

Shao, Q.-M. (1995). Maximal inequality for partial sums of $\rho$-mixing sequences. Ann. Probab., 23, 948-965.

Stoker, T.M. (1986). Consistent estimation of scaled coefficients. Econometrica, 54, 14611481.

Stoker, T.M. (1989). Tests of additive derivative constraints. Rev. Econom. Stud., 56, 535552. 
Stoker, T.M. (1991), Equivalence of Direct, Indirect and Slope Estimators of Average Derivatives, Nonparametric and Semiparametric Methods in Econometrics and Statistics. W.A. Barnett, J. Powell and G. Tauchen, eds., Cambridge University Press.

Türlach, B.A. (1994). Fast implementation of density-weighted average derivative estimation. Computationally Intensive Statistical Methods, 26, 28-33.

White, H. and Domowitz, I. (1984). Nonlinear Regression with Dependent Observations. Econometrica, 52, 143-162.

Vidakovic, B. (1999). Statistical Modeling by Wavelets. John Wiley \& Sons, Inc., New York, $384 \mathrm{pp}$.

Zhengyan, L. and Lu, C. (1996). Limit Theory for Mixing Dependent Random Variables. Kluwer, Dordrecht. 